\title{
Improving national surveillance of Lyme neuroborreliosis in Denmark through electronic reporting of specific antibody index testing from 2010 to 2012
}

R B Dessau (ramd@regionsjaelland.dk)², L Espenhain2, K Mølbak², T G Krause ${ }^{2}$, M Voldstedlund ${ }^{2}$

1. Department of Clinical Microbiology, Slagelse Sygehus, Slagelse, Denmark

2. Department of Infectious Disease Epidemiology, Statens Serum Institut, Copenhagen, Denmark

Our aim was to evaluate the results of automated surveillance of Lyme neuroborreliosis (LNB) in Denmark using the national microbiology database (MiBa), and to describe the epidemiology of laboratory-confirmed LNB at a national level. MiBa-based surveillance includes electronic transfer of laboratory results, in contrast to the statutory surveillance based on manually processed notifications. Antibody index (AI) testing is the recommend laboratory test to support the diagnosis of LNB in Denmark. In the period from 2010 to 2012, 217 clinical cases of LNB were notified to the statutory surveillance system, while 533 cases were reported Al positive by the MiBa system. Thirty-five unconfirmed cases (29 Al-negative and 6 not tested) were notified, but not captured by MiBa. Using MiBa, the number of reported cases was increased almost 2.5 times. Furthermore, the reporting was timelier (median lag time: 6 vs 58 days). Average annual incidence of Al-confirmed LNB in Denmark was 3.2/100,000 population and incidences stratified by municipality ranged from none to above $10 / 100,000$. This is the first study reporting nationwide incidence of LNB using objective laboratory criteria. Laboratory-based surveillance with electronic data-transfer was more accurate, complete and timely compared to the surveillance based on manually processed notifications. We propose using Al test results for LNB surveillance instead of clinical reporting.

\section{Introduction}

Borrelia species, known to cause Lyme borreliosis, are collectively known as Borrelia burgdorferi sensu lato. $B$. burgdorferi sensu stricto is the cause of Lyme borreliosis in North America, whereas B. afzelii and B. garinii cause most European cases [1]. Differences in the clinical presentation of Lyme borreliosis in North America and Europe are ascribed to differences in the predominant species. One important systemic manifestation of
Lyme borreliosis is Lyme neuroborreliosis (LNB), which is a clinically characteristic neurological syndrome. Notably LNB is much more common in Europe where $B$. garinii is frequently the causative agent. In Denmark $B$. afzelii has been isolated from patients with erythema migrans and $B$. garinii from patients with LNB [2]. The disease is transmitted by the hard tick (Ixodes ricinus or $I$. persulcatus). The abundance of $I$. ricinus is determined by complex interaction with many factors including wildlife, geography and climate [3]. According to the clinical European case definition developed by the European Federation of Neurological Societies (EFNS), and also written stated by the European Study group for Lyme Borreliosis (ESGBOR) under the European Society for Clinical Microbiology and Infectious Diseases, demonstration of intrathecal antibody production (antibody index, Al) is essential for the diagnosis of LNB $[4,5]$. In patients with short duration of clinical disease, antibody response may be weak or absent [5]. The original development of a new assay and diagnostic criteria to diagnose patients with LNB with Al in Denmark was described by Klaus Hansen and co-workers [6-9] and this assay has been found sensitive and specific [5,8-10].

In Denmark, surveillance of LNB started in 1991 when the Danish Health and Medicines Agency decided to make LNB a mandatory clinical notifiable disease to Statens Serum Institut (SSI), as LNB was considered to be the most common severe clinical manifestations of Lyme borreliosis. LNB is relevant for surveillance, as it is of public health interest to follow trends due to climate change or altered outdoor behaviour in order to adopt appropriate preventive measures. The incidences of the other disseminated manifestations of Lyme borreliosis such as acrodermatitis, carditis, Lyme arthritis and lymphocytoma are not known in Denmark. The incidence of patients suspected of these 


\section{FIGURE 1}

Flowchart of patients suspected for Lyme neuroborreliosis (LNB) included in the study, Denmark, 2010-2012 (n=13,929 suspected patients)
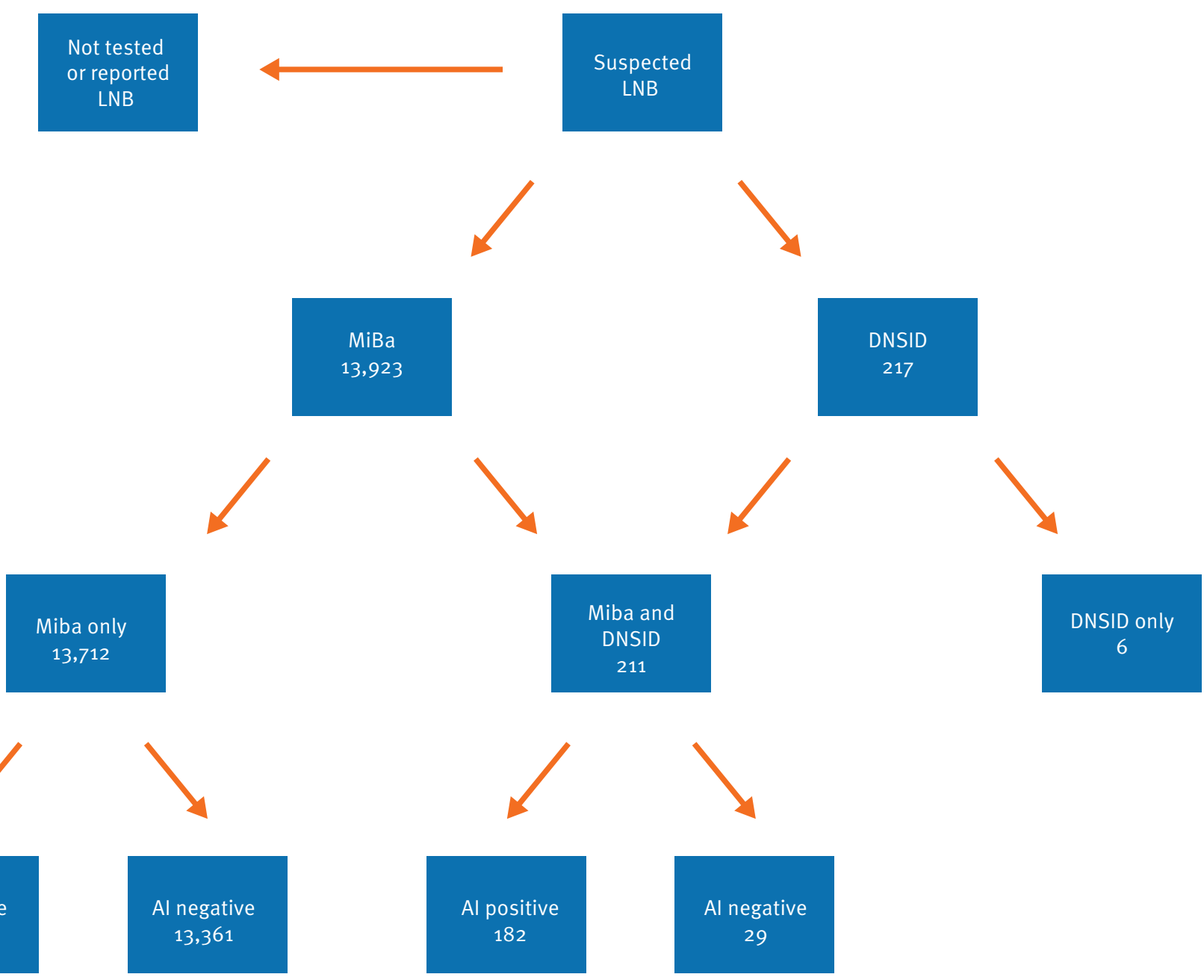

Al: antibody index; DNSID: Danish notification system for infectious diseases; MiBa: Danish microbiology database

other manifestations was however 47/100.000, but a low seropositivity rate, similar to healthy controls, was found in consecutive patients seen in general practice, which suggests a low incidence and poor positive predictive value of serology in most cases [11].

The current statutory Danish notification system for infectious diseases (DNSID) is based on collection of paper forms completed by the physician treating the patient; the forms are sent by mail to the Department of Infectious Disease Epidemiology at SSI and the Regional Medical Officer of Health. SSI sends reminders to the clinicians if intrathecal antibody production has been detected by the SSI laboratory and no notification has been received within a certain timeframe. From 2011 to 2012, 44\% of the notifications was received only after a reminder had been sent. Due to increased testing at the regional microbiological laboratories, SSI is responsible for a decreasing fraction of the Al tests in Denmark, and therefore the current surveillance system for LNB is neither timely nor complete, even considering the sending of reminders.

The Danish microbiology database ( $\mathrm{MiBa}$ ) has recently been developed and has since 2010 received electronic copies of all reports from all Danish departments of clinical microbiology [12]. MiBa has several objectives including improving the national laboratory-based surveillance [10]. The aim of the present study was to assess MiBa for laboratory-based surveillance of LNB, including a comparison with the prevailing system based on manually-processed clinical notifications. Furthermore, we use the data from MiBa to describe the epidemiology of laboratory-confirmed LNB in Denmark.

\section{Methods}

The study period was from January 2010 to December 2012 and the study population was the population of Denmark. 


\section{FIGURE 2}

Annual numbers of Lyme neuroborreliosis cases identified in the Danish microbiology database (MiBa) from 2010 to 2012 and annual numbers of notified cases, Denmark, 2000-2012

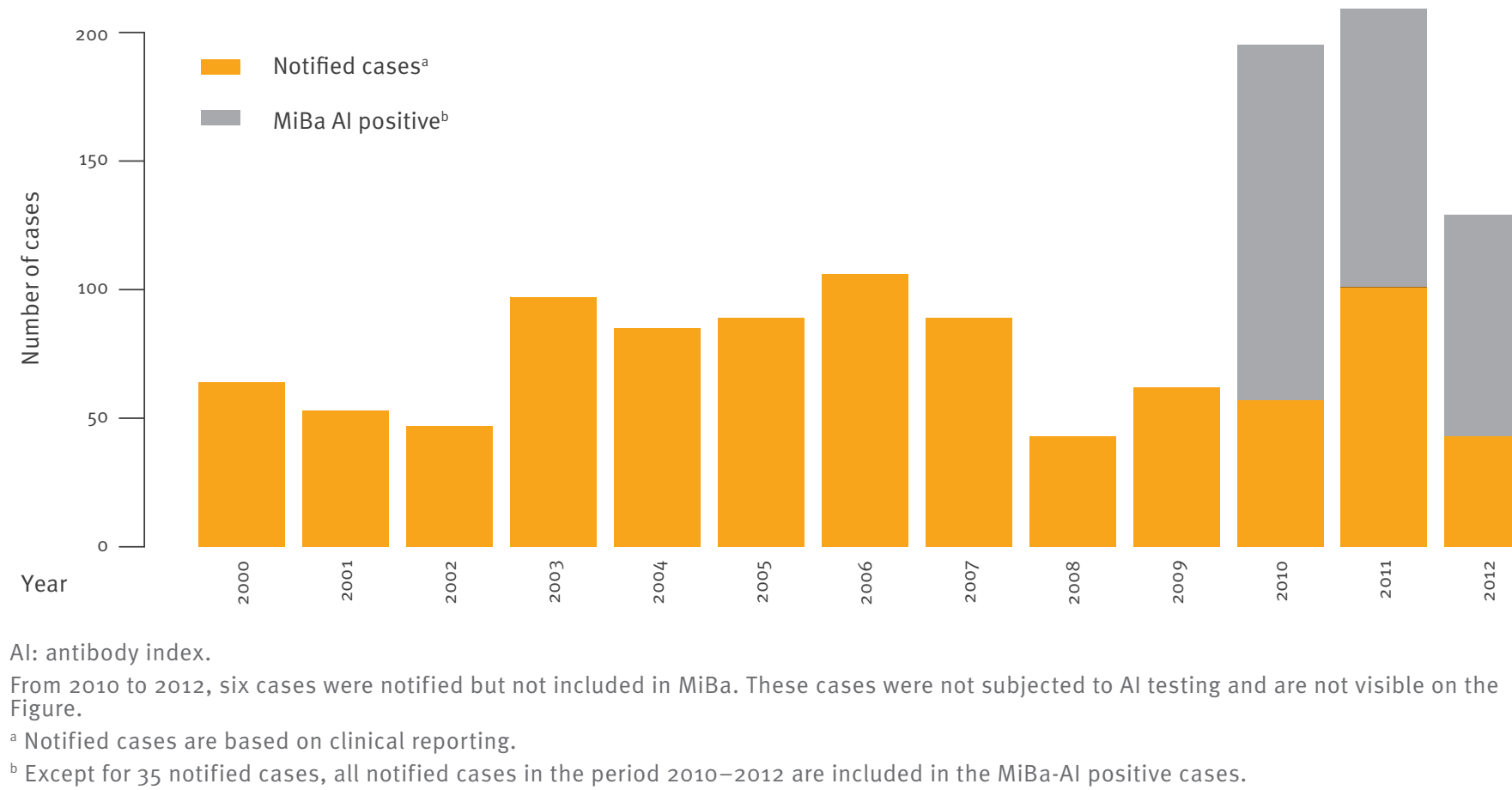

\section{Data sources}

The statutory Danish notification system for infectious diseases

DNSID is a case-based database including information on the civil registration number (CPR number) of the case, date of disease-onset, symptoms, laboratory results as well as sequelae. The CPR number is a unique identification number given to each person living in Denmark.

Both confirmed and probable cases (see below) were included in the DNSID dataset extracted for the present study, as both, so far, have been included in the national surveillance.

The Danish microbiology database

MiBa receives real-time electronic copies of all reports from all Danish departments of clinical microbiology [12]. The data model and basic principles have been described previously $[12,13]$. Within MiBa local codes are automatically mapped to national shared codes before data extraction. All reports include the CPR number of the patient.

In Denmark a total 11 laboratories performed Al tests. At the time of the study, nine of these reported to MiBa through their microbiology laboratory information systems. The two remaining laboratories included one biochemistry laboratory that did not report to $\mathrm{MiBa}$, and one laboratory that had technical problems with the transfer protocol during the first two years. To obtain complete nationwide data, data on Al test results were acquired directly from the two latter laboratories and merged with the MiBa data. This merged dataset is referred to as the MiBa dataset hereafter.

The Danish civil registration system

Using the CPR number information on age, sex, address, and municipality was obtained. Information on population size was obtained from Statistics Denmark (www.dst.dk). To represent the middle of the study period, data was retrieved for the first quarter of 2011, were Denmark had a total population of 5.56 million people.

\section{Definitions and data management}

The statutory case definition for LNB in DNSID is as follows:

- Confirmed case: patient with clinical symptoms con sistent with LNB and a positive Al test.

- Probable case: patient with clinical symptoms consistent with LNB and borrelia antibodies in serum.

- Concerning the probable cases the $\mathrm{Al}$ is either negative or not done, but the detection of serumantibodies is required.

- Case definition for LNB in MiBa:

- A patient with one or more Al tests positive for Borrelia IgG or IgM antibodies or both. 


\section{FIGURE 3}

Monthly numbers of antibody index (AI) positive Lyme neuroborreliosis (LNB) cases and average annual incidence of cases and patients tested, Denmark, 2010-2012

A Number of Al positive LNB cases

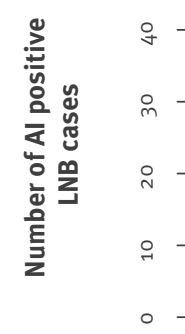

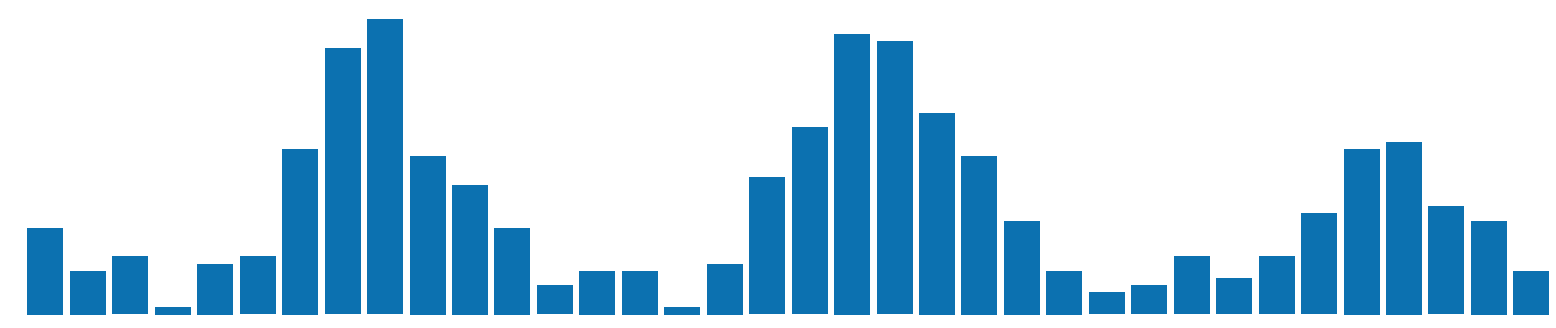

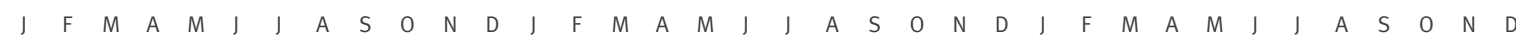

Month from January 2010 to December 2012

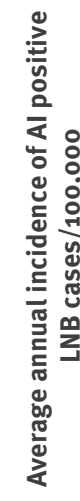

B Average annual incidence of Al positive LNB cases/100,000 population stratified by age group

$\left.\begin{array}{ll}0 & \\ \text { n } & - \\ v & - \\ m & - \\ N & - \\ - & - \\ 0 & \end{array}\right]$
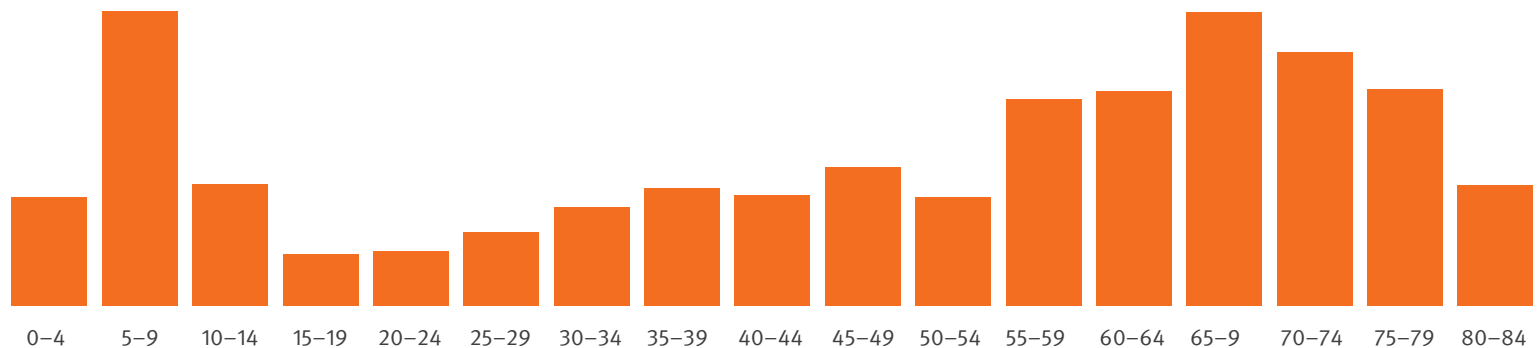

Age groups in years

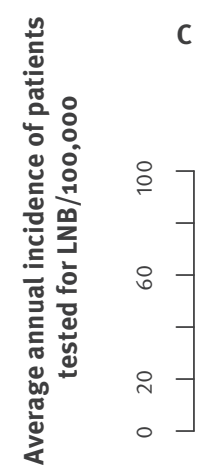

C Average annual incidence of patients tested for LNB/100,000 population stratified by age group

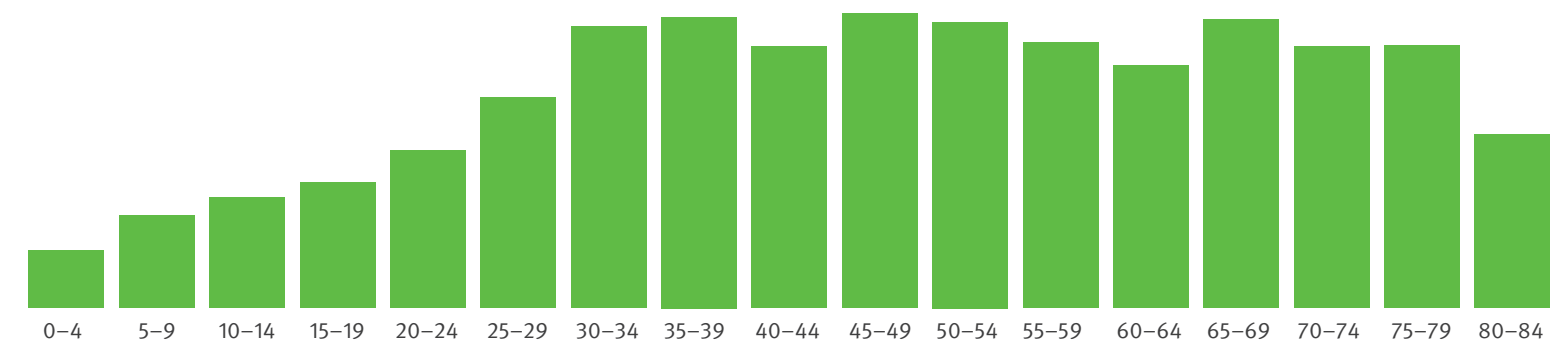

Age groups in years 


\section{FIGURE 4}

Three year average of annual incidence of Lyme neuroborreliosis antibody index-positive cases, according to municipalities, Denmark, 2010-2012

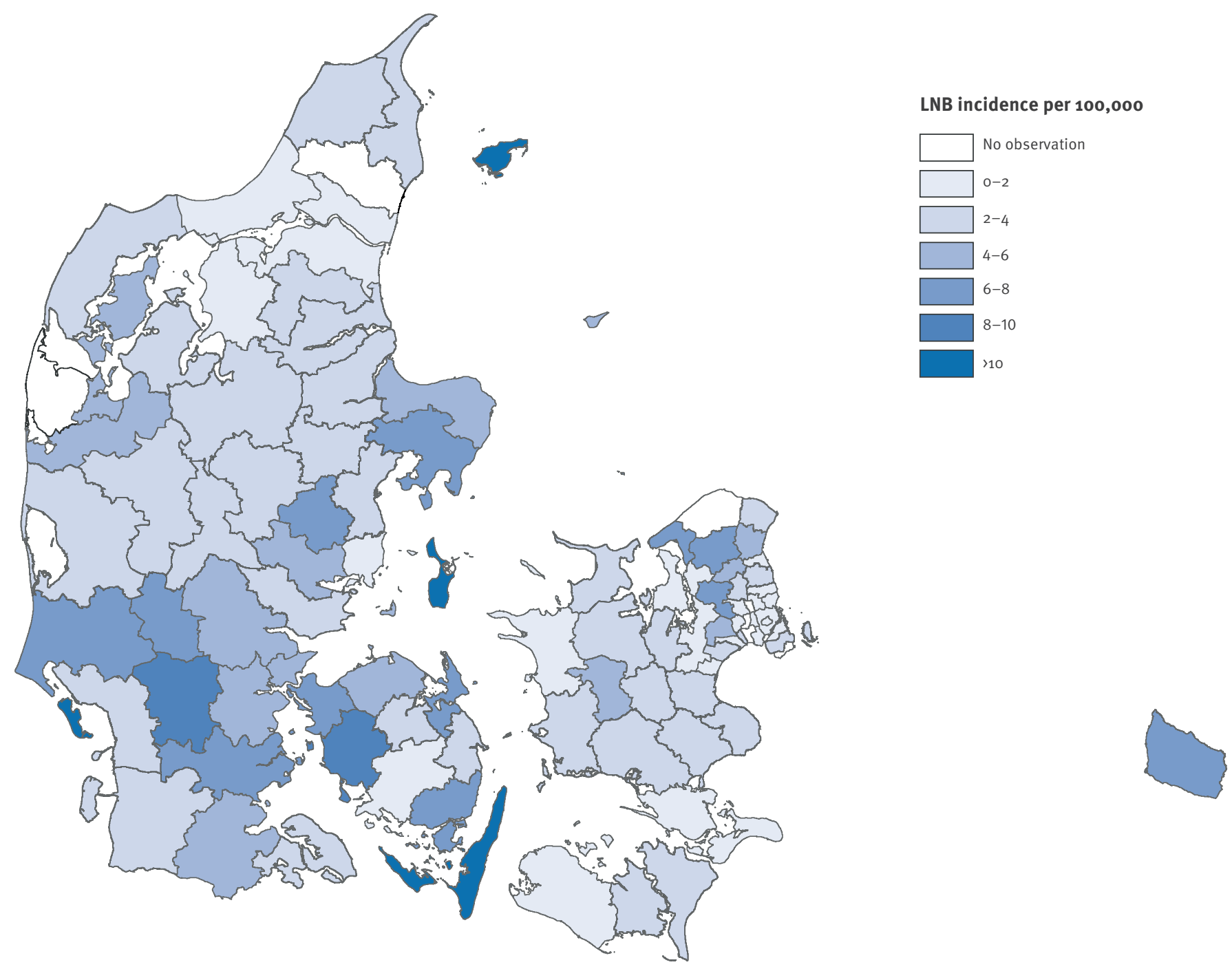

Data management

Patients residing in Greenland and patients with temporary CPR numbers, including foreign travellers, were excluded from all datasets.

The raw MiBa dataset included results from more than one test report per patient. This raw dataset was transformed into a dataset with only one record per CPR number (case) according to the following rules:

Each patient was classified as IgG positive, IgM positive or both IgG-and-IgM positive, based on the accumulated Al-results of one or more reports. If a test result was stated as inconclusive it was considered as negative. A patient was only included once during the three year study period with the first positive test as a case-defining event. A patient with only negative Al tests was included as a LNB negative patient with the date of the first test performed. The total number of tests performed per patient was registered. The age was calculated as age at the date of disease.
Reports on tests that for some reason were not performed (e.g. sample tube broken during transport) were excluded from the dataset.

Intrathecal antibody production

For this study, with the purpose of surveillance, the conclusion by the laboratory in the report was considered valid regardless of the type of assay and method of index calculation. All laboratories except one used an assay based on native purified flagella antigen (IDEIA LNB IgG/IgM assay, Oxoid, Cambridgeshire, United Kingdom). The index calculation is specified by the manufacturer with the formula: Index=(ODcsf) ODserum)*(ODcsf - -ODserum), where ODcsf and ODserum is the optical absorbance in the cerebrospinal fluid and the serum, respectively. An index above 0.3 was considered as positive. If the absolute absorbance (abs.) in the spinal fluid was below 0.150 the result was negative in any case. The assay is a capture 


\section{FIGURE 5}

Three year average of antibody index-tested patients for Lyme neuroborreliosis per 100,000 population according to municipalities, Denmark, 2010-2012

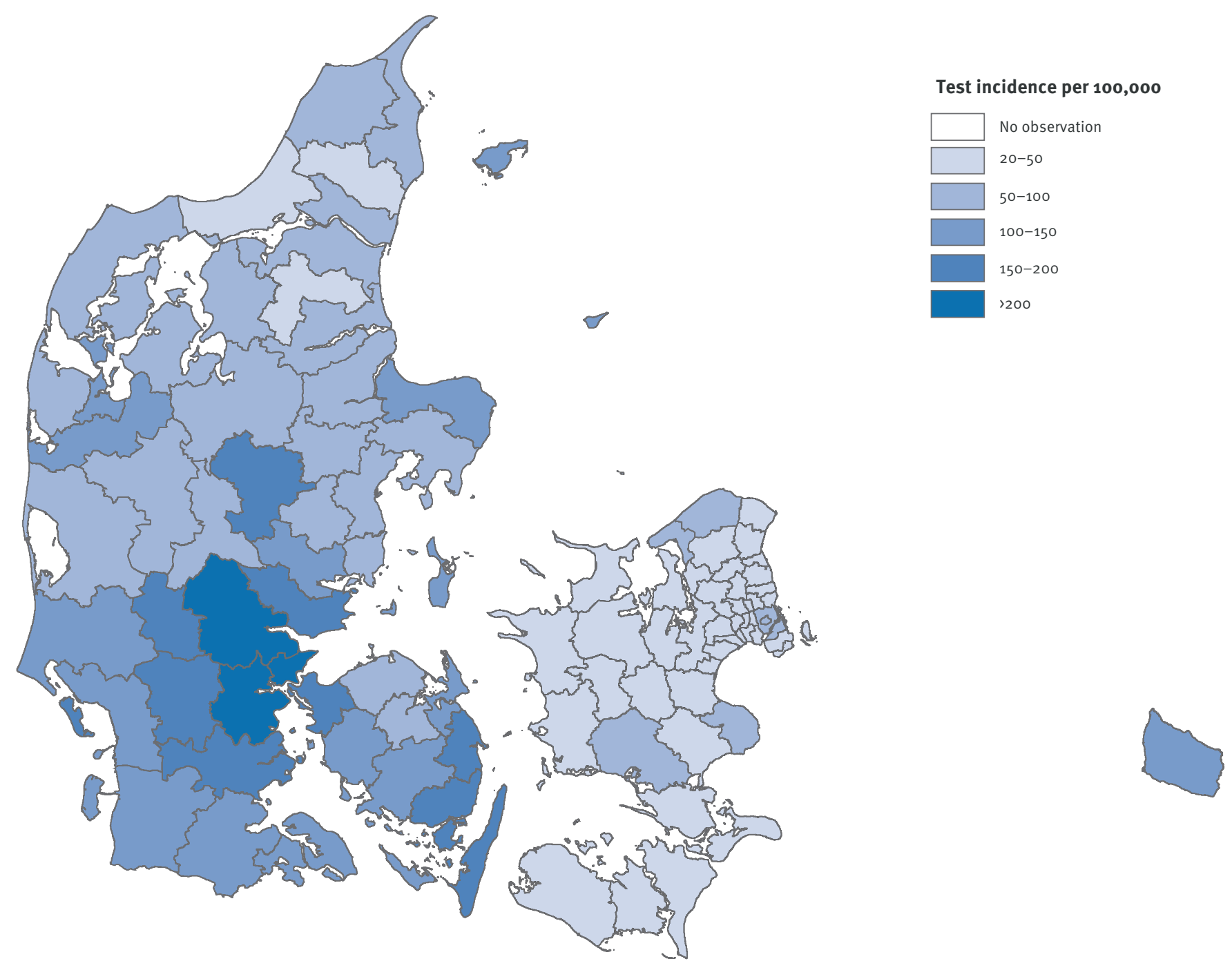

enzyme-linked immunosorbent assay (ELISA) thus the relative abundance of Borrelia specific antibodies compared with the total IgG is important for a positive result and measurements of total IgG are not needed in the algorithm.

\section{Data linkage}

Data from DNSID, MiBa, and the Danish civil registration system was linked using the CPR number.

\section{Timeliness}

The timeliness of the two systems was calculated as a technical time lag in days from sampling date to the date, where information on the case was received at $\mathrm{SSI}$, either in the form of a notification or when the test result in MiBa was available for data extraction. From the DNSID surveillance only confirmed cases were included (182 observations). For the MiBa surveillance Al positive LNB cases were included.

\section{Statistical methods}

Data management was performed using SAS (SAS Institute Inc., Cary, North Carolina, United States) and statistical analyses were performed in $\mathrm{R}$ [14] using chi-squared tests and confidence intervals for proportions. Total number of tested patients and LNB cases were stratified by age group, sex, and municipality of residence and presented per 100,000 population. Also annual (cumulative) incidences were calculated per 100,000 population. For the geographical presentation of data QGIS (version 2.4.0) was used.

\section{Ethical considerations}

This study was approved by the Danish data protection authority as part of a general permission for performing surveillance studies (registration number 200854-0472) and falls within the regulatory community framework for the national surveillance in Denmark 


\section{TABLE 1}

Number of patients tested for Lyme neuroborreliosis (LNB) in the Danish microbiology database and number of notified cases of LNB in Denmark, 2010-2012

\begin{tabular}{|c|c|c|c|}
\hline \multirow{2}{*}{ Year } & \multicolumn{2}{|c|}{ MiBa $^{\mathrm{a}}$} & DNSID \\
\cline { 2 - 4 } & Tested $^{\mathrm{b}}$ & Cases $^{\mathrm{c}}(\%$ positive) & Notified cases $^{\mathrm{d}}$ \\
\hline 2010 & 4,347 & $195(4.5)$ & 57 \\
\hline 2011 & 4,957 & $209(4.2)$ & 101 \\
\hline 2012 & 4,619 & $129(2.8)$ & 59 \\
\hline Total & 13,923 & $533(3.8)$ & $217^{\mathrm{e}}$ \\
\hline
\end{tabular}

DNSID: Danish notification system for infectious diseases; MiBa: Danish microbiology database.

a Numbers are based on the MiBa-case dataset.

b Number of patients tested by LNB specific antibody index tests. Number of cases which are antibody index positive for borrelia IgM, IgG or both.

d Number of cases clinically reported on notifications.

Of the total 217 notified patients in DNSID six were probable cases, which were not subjected to antibody index testing and 29 had a negative antibody index test. The remaining 182 notified patients had a positive antibody index test.

(the National Board of Health Statutory Order on Physicians' Notification of Infectious Diseases).

\section{Results}

\section{Comparison between $\mathrm{MiBa}$ and the mandatory} notification system

According to MiBa from 2010 to 2012 a total of 13,923 patients were tested by Al and LNB was confirmed in 533 (4\%) patients (Table 1 ). Of these 172 (32\%) were only IgG positive, 103 (19\%) only IgM positive and 258 $(48 \%)$ cases were found positive in both $\lg M$ and $\lg G$. By contrast, in DNSID only 217 patients were notified as LNB in this period (Figure 1, Table 1). Among the 217 DNSID patients, Al tests were positive for 182, while 35 patients were either Al negative (29 patients) or not tested by Al (6 patients).

When data from DNSID and MiBa were linked, we found that of 13,923 tested patients in MiBa, 211 were also in DNSID and 182 (83\%) of these were Al positive (Table 1, Figure 1).

Thus in MiBa 351 cases of LNB were identified, which had not been registered by the national surveillance. These cases were compared with the notified cases in Table 2. Among the $351 \mathrm{MiBa}$ cases not notified 65 $(19 \%)$ were children (0-15 years of age) and this was significantly lower compared with 64 children (29\%) of 217 cases notified in DNSID (relative risk (RR): 0.63; 95\% confidence interval $(\mathrm{Cl}): 0.47-0.84)$. Also twelve (34\%) children were found among the 35 cases notified where Al was either negative or not performed, which may be compared with 65/351 (19\%) which were
Al positive but not notified in DNSID (RR: $1.8 ; 95 \% \mathrm{Cl}$ : 1.1-2.9).

The regional distribution of LNB cases was different between notified and unnotified cases identified in MiBa.

Since 2000 the average number of notified cases was 73 per year (Figure 2), corresponding to an annual incidence of LNB of 1.3 cases/100,000 population. The average annual number of notified cases from 2010 to 2012 was 67 and thus comparable to the previous years. In the same period, the average number of cases identified in MiBa was 178 per year, which yielded an annual incidence, of 3.2/100,000.

The median time lag from sampling date to reception of the notification by SSI was 58 days (range: 6-613), whereas the median time lag from sampling date to availability for data extraction in MiBa was five days (range: 1-106). The reports with more than 20 days delay were due to transfer of samples from some local laboratories, for testing at the reference laboratory at SSI.

\section{Descriptive epidemiology of Lyme neuroborreliosis in Denmark}

The data from MiBa show a clear seasonal variation of Al-confirmed LNB cases, with lower numbers in the period between February to May and peaks in August and September (Figure $3 \mathrm{~A}$ ). Also the average number of patients tested for $\mathrm{Al}$ exhibited seasonal variation with lowest number in April $(n=278)$ and highest in August $(n=468)$. The average monthly percentage of positive test results among patients tested ranged from $1.2 \%$ in April to $7.4 \%$ in September. Thus during winter and early spring the diagnostic yield is low.

The age-specific annual incidences of LNB were highest among children (5-10 years) and the older age groups (55 to 79 years-old) with a peak at 65 to 69 years of age (Figure $3 \mathrm{~B}$ ). In contrast, the average annual cumulative age-specific incidence of patients being tested, increased with age until 30 years of age, over which the incidence of testing was stable and caabout 100/100,000 population (Figure 3C).

\section{Geographical distribution}

For the geographical distribution of LNB, the DNSID data were not used as it they wereas considered unreliable due to differences found in reporting frequency between regions (Table 2). Based on MiBa data, the average annual incidence of LNB stratified by municipality ranged from none to more than 10/100,000 (Figure 4). 'Hot spots' were found in northern Zealand, Funen and parts of southern Jutland, and interestingly in many of the 'smaller' islands (Bornholm, Læs $\emptyset$, Sams $\emptyset$, Langeland, Æ Ærø and Fan $\varnothing$ ). These results were not adjusted for differences in age and sex distribution between municipalities. 
TABLE 2

Comparison of the 351 Lyme neuroborreliosis cases identified in the Danish microbiology database (MiBa) but not notified, with the 217 notified cases, and annual incidences of all antibody index positive cases in MiBa, Denmark, 2010-2012

\begin{tabular}{|c|c|c|c|c|c|}
\hline \multirow{2}{*}{$\begin{array}{l}\text { Characteristics of } \\
\text { cases }\end{array}$} & \multicolumn{2}{|c|}{ Notified cases of neuroborreliosis } & \multirow{2}{*}{$\begin{array}{l}\text { MiBa cases not } \\
\text { notified }\end{array}$} & \multirow{2}{*}{$\begin{array}{l}\text { MiBa cases not notified } \\
\text { vs notified cases } \\
\text { RR }(95 \% \mathrm{Cl})\end{array}$} & \multirow{2}{*}{$\begin{array}{l}\text { All cases in MiBa: average } \\
\text { annual incidence per } \\
100,000 \text { population }\end{array}$} \\
\hline & Positive Al & $\begin{array}{l}\text { No result or negative } \\
\text { Al result }\end{array}$ & & & \\
\hline \multicolumn{6}{|l|}{ Age group in years } \\
\hline $0-15$ & 52 & 12 & 65 & $\begin{array}{c}0.63 \\
(0.47-0.84)\end{array}$ & $3 \cdot 7$ \\
\hline $16-64$ & 90 & 17 & 188 & $\begin{array}{c}1.08 \\
(0.92-1.29) \\
\end{array}$ & 2.6 \\
\hline$\geq 65$ & 40 & 6 & 98 & $\begin{array}{c}1.32 \\
(0.97-1.80)\end{array}$ & 5.0 \\
\hline \multicolumn{6}{|l|}{ Sex } \\
\hline Male & 106 & 18 & 200 & $\begin{array}{c}1.08 \\
(0.94-1.24) \\
\end{array}$ & $3 \cdot 7$ \\
\hline Female & 76 & 17 & 151 & $\begin{array}{c}1.00 \\
(0.82-1.22) \\
\end{array}$ & 2.7 \\
\hline \multicolumn{6}{|l|}{ Region of residence } \\
\hline Capital & 76 & 18 & 39 & $\begin{array}{c}0.26 \\
(0.18-0.36) \\
\end{array}$ & 2.3 \\
\hline Zealand & 19 & 2 & 46 & $\begin{array}{c}1.35 \\
(0.84-2.20) \\
\end{array}$ & 2.6 \\
\hline Southern Denmark & 44 & 7 & 138 & $\begin{array}{c}1.67 \\
(1.28-2.21) \\
\end{array}$ & 5.1 \\
\hline Middle Jutland & 23 & 1 & 106 & $\begin{array}{c}2.73 \\
(1.83-4.12) \\
\end{array}$ & 3.4 \\
\hline Northern Jutland & 20 & 6 & 15 & $\begin{array}{c}0.36 \\
(0.20-0.65)\end{array}$ & 2.0 \\
\hline Unknown ${ }^{\mathrm{a}}$ & 0 & 1 & 7 & NA & NA \\
\hline Total & 182 & 35 & 351 & 1.00 & 3.2 \\
\hline
\end{tabular}

Al: antibody index; $\mathrm{Cl}$ : confidence interval; $\mathrm{NA}$ : not applicable; RR: relative risk.

a Unknown address of residence.

The geographical distribution of LNB (Figure 4) did not just reflect differences in frequency of testing (Figure 5). For example, absence of cases in three municipalities was not explained by lack of testing. Also municipalities with the highest incidence of Al testing (Figure 5) did not have the highest incidence of LNB cases.

\section{Discussion}

We compared the existing surveillance system, DNSID, based on manually processed notifications with a new laboratory-based system, MiBa, automatically compiling all $\mathrm{Al}$ test performed on a national level. In spite of fundamental differences between the systems, the data are comparable since the case definitions for both the MiBa-based-system and for confirmed cases in the DNSID-based surveillance rely on the Al test for LNB. However, the Danish clinical guideline on Lyme borreliosis recommends, not only a positive $\mathrm{Al}$, but also a lumbar puncture with leucocytosis, for the diagnosis of LNB (www.dskm.dk). In MiBa there is no access to spinal-fluid leucocyte counts. Leucocytosis in the spinal fluid is an important marker of active disease and, if absent, a positive Al probably indicates past infection. In an earlier study of 3,756 Al tests, the sensitivity was estimated to be $88 \%$ and specificity $99.7 \%$ for LNB
$[8,9,15]$. Of $125 \mathrm{Al}$ positive patients, seven patients did not show leucocytosis, indicating previous LNB. Thus, a case definition solely based on Al testing, would provide an adequate specificity for surveillance in a Danish context, where previous LNB is rare. The risk of reporting past infections as active LNB would be ca $6 \%$. We therefore consider this concern as less important compared with the advantages of an automated system.

The existing surveillance includes notifications of both probable cases as well confirmed cases (Al-positive). On average 12 patients (35 cases/3 years) were notified as probable cases per year; these were not captured by $\mathrm{MiBa}$. The probable cases were more frequent in children less than 16 years-old (Table 2), thus if these 12 were all true cases, some children would be missed by a surveillance based on laboratory reporting of Al positive results only. In addition, there could be a number of patients treated for LNB on a putative diagnosis, which were neither tested nor reported. However, this is not limitation for surveillance as this group of patients may contain misclassified non-LNB patients as well. A surveillance of $L N B$ based on $A$ l results would have a low risk of including misclassified cases. We assume that a lumbar puncture is only performed, when neurological 
symptoms and objective findings justify this invasive procedure. Thus testing, without relevant indication, which could lead to a low positive predictive value, is considered rare.

MiBa receives in principle all test reports from all departments of clinical microbiology. However, a few tests for infectious diseases are for historical reasons still reported by a biochemistry laboratory. This biochemistry laboratory and one department of clinical microbiology were unable to report electronically to $\mathrm{MiBa}$ in the study period. Prospectively, MiBa will be complete on LNB Al reports at the national level. The automated transfer of all reports frees physicians and laboratories from active reporting; and makes surveillance independent of local healthcare personnel remembering to do the notification. The present study demonstrated underreporting as only $34 \%$ of the 533 Al positive LNB were notified. This probably reflects both the workload associated with filling in and sending paper forms and uncertainty on whether LNB is notifiable or not. The 533 cases over three years, might be slightly underestimated, as we did not take into account that it is possible to have more than one episode of LNB during this period.

The present study described an improved system for surveillance of LNB based on objective criteria. It also provided an important insight into the epidemiology of LNB in Denmark. The incidence of LNB in Denmark was found to be more than twice as high as previously estimated. An average annual incidence of 3.2/100,000 in Denmark is comparable to a one year Swedish study from May 1992 to April 1993 in which the incidence of LNB with lymphocytic pleocytosis was found to be $2 / 100,000$ [16]. The incidence of Al positivity was not reported in this study. In the Würzburg region of Germany a yearly incidence for LNB of 3/100,000 may be calculated from the study from May 1996 to April 1997, however the Al was not specified as part of the case definitions [17]. In Norway, surveillance of LNB is based on a broader case definition comprising clinical symptoms with IgM antibodies in serum or spinal fluid or evidence of intrathecal production. The Norwegian Public Health Institute reports an average incidence of 6/100,000 per year (www.msis.no). However, including cases based on IgM antibodies in serum alone may confer problems with specificity and risk of overreporting. LNB is rare in the US with an incidence of $0.07 / 100,000$ per year (www.cdc.org). This is due to the occurrence of $B$. burgdorferi sensu stricto in the US and the absence of $\mathrm{B}$. garinii, which is the principal cause of LNB in Europe [18]. In 2013 the reported annual incidence of other causes of bacterial meningitis reported in Denmark was 3.0/100,000 and of these, pneumoccal meningitis represented 1.4/100,000 [19]. Thus LNB with an incidence of 3.2/100,000 per year was the most frequent bacterial cause of infection affecting the brain.
LNB is a relatively acute infection and this was reflected in the seasonal variation found in the present study. The number of LNB cases peaked in August and September, in line with a previous Danish study on LNB [8].

An advantage of the MiBa-based surveillance is that it also includes data on negative test results. This makes it possible to study healthcare practices including testing activity on an individual level in a defined population and calculate positive rates. We found that testing for LNB is common and that $96 \%$ were negative. During the late winter and the early spring, the diagnostic yield is very low. The low positivity rates suggest that symptoms suggestive of LNB are caused by other diseases, reflecting the non-specific nature of symptoms compatible with LNB.

Interestingly, the population-based LNB incidences stratified by age, sex and geography showed a different pattern compared with the incidences of patients being tested. Testing activity was highest in the age group 30 to 79 years, whereas the annual incidence of LNB peaked in childhood and again in the older age groups (55 to 79 years). Children are presumably exposed when they are playing, and a Dutch study found that people over 60 years were often bitten by ticks in their gardens [20].

The geographical distribution of Al-confirmed LNB has not been described previously in Denmark. In the present study, the results from MiBa indicated that annual incidence varied substantially across the country from zero to more than $10 / 100,000$. The finding of hot spots on smaller islands has also been described for the Finish archipelago forming the region of Åland in the Baltic Sea [21]. Three Danish municipalities had no LNB cases; this absence could not be explained by lack of tested persons as the incidence of tested persons was 20-100/100,000 (Figure 4 and 5). Compared with the statutory notifications, the MiBa-based surveillance showed a different geographical distribution of LNB. The statutory system is biased by regional differences in notification rates; this bias is in part explained by SSI only sending reminders for missing notifications, based on laboratory results performed at SSI, which mainly received samples from the Capital region. This was not the case if testing was done at a regional laboratories because the epidemiological department at SSI then would be unaware of a possible positive AI. One limitation in the description of the geographical distribution is that we only have information on place of residence, which is not necessarily the place of exposure to tick bites.

Whereas the statutory notification system asks for information on clinical manifestation and sequelae, this information is not available in MiBa. In any case, the reporting of this additional clinical information was too incomplete to be useful (data not shown). Also asking for sequelae does not make sense in a reporting 
system concerning acute disease as this requires a longer follow up. However, as the LNB cases in MiBa have a unique identifier that allows for linkage with other health registries, it will be possible to further explore risk factors for developing LNB, clinical manifestations and long-term sequelae in ad hoc projects. It will also be possible to compare testing for LNB with testing for other tick-borne diseases.

In time of writing, MiBa has accumulated data for four years, and procedures for data cleaning, analysis and aggregation are being automatised. Soon we will be able to calculate baselines and analyse for trends.

It may be relevant also to have surveillance in place for other clinical manifestations of Lyme borreliosis such as erythema migrans in order to estimate the burden of Lyme borreliosis. This may be possible in the future through data-capture from general practitioners' databases and the national patient register, as active reporting from clinicians would be difficult to motivate and organise. Other systemic manifestations in Denmark are rare and have been shown not to contribute significantly to the burden of Lyme borreliosis in clinical practice [11]. It was shown that consecutive patients with suspected Lyme arthritis had the same level of IgG seropositivity as had been found in Danish blood donors, indicating that the positive predictive value of the serology was negligible [11]. Thus being a rare unspecific clinical syndrome lacking a specific diagnostic test, Lyme arthritis is not a candidate for national surveillance.

\section{Conclusion}

In the present study, we found that a surveillance of LNB based on data from MiBa was more timely and more complete compared with the statutory surveillance based on manually processed notifications. We propose electronic reporting using the $A I$ to replace clinical reporting as the basis of future LNB surveillance.

\section{Acknowledgements}

We would like to thank all Danish Departments of Clinical Microbiology collaborating in the MiBa Project and for contributing with their data. We also thank the department of Clinical Biochemistry, Regional Hospital Central Jutland and the Reference laboratory at SSI for sharing separate datasets. We thank Catherine Collin Bjerre for her contribution in the start-up phase of the study. The Danish Microbiology Database is funded by the Danish Ministry of Health.

\section{Conflict of interest}

None declared.

\section{Authors' contributions}

$R$ Dessau has performed the statistical data analysis and drafted the first versions. Marianne Voldstedlund has performed data extraction with L Espenhain. L Espenhain has created the incidence maps of Denmark. All authors have contributed to the conception of the study and the revisions of the final manuscript.

\section{References}

1. Stanek G, Reiter M. The expanding Lyme Borrelia complex-clinical significance of genomic species? Clin Microbiol Infect. 2011;17(4):487-93. http://dx.doi.org/10.1111/j.14690691.2011.03492.x PMID:21414082

2. Lebech AM, Hansen K, Wilske B, Theisen M. Taxonomic classification of 29 Borrelia burgdorferi strains isolated from patients with Lyme borreliosis: a comparison of five different phenotypic and genotypic typing schemes. Med Microbiol Immunol (Berl). 1994;183(6):325-41. http://dx.doi.org/10.1007/ BFo0196683 PMID:7541107

3. Medlock JM, Hansford KM, Bormane A, Derdakova M Estrada-Peña A, George JC, et al. Driving forces for changes in geographical distribution of Ixodes ricinus ticks in Europe. Parasit Vectors. 2013;6(1):1. http://dx.doi.org/10.1186/17563305-6-1 PMID:23281838

4. Mygland A, Ljøstad U, Fingerle V, Rupprecht T, Schmutzhard E, Steiner I; European Federation of Neurological Societies. EFNS guidelines on the diagnosis and management of European Lyme neuroborreliosis. Eur J Neurol. 2010;17(1):816, e1-4. http://dx.doi.org/10.1111/j.1468-1331.2009.02862.x PMID:19930447

5. Stanek G, Fingerle V, Hunfeld KP, Jaulhac B, Kaiser R, Krause A, et al. Lyme borreliosis: clinical case definitions for diagnosis and management in Europe. Clin Microbiol Infect. 2011;17(1):69-79. http://dx.doi.org/10.1111/j.14690691.2010.03175.x PMID:20132258

6. Hansen K, Rechnitzer C, Pedersen NS, Arpi M, Jessen O. Borrelia meningitis in Denmark. Zentralbl Bakteriol Mikrobiol Hyg [A]. 1987;263(3):348-50. http://dx.doi.org/10.1016/So1766724(87)80090-1 PMID:3591087

7. Hansen K, Lebech AM. Lyme neuroborreliosis: a new sensitive diagnostic assay for intrathecal synthesis of Borrelia burgdorferi--specific immunoglobulin G, A, and M. Ann Neurol. 1991;30(2):197-205. http://dx.doi.org/10.1002/ana.410300212 PMID:1897911

8. Hansen K, Lebech AM. The clinical and epidemiological profile of Lyme neuroborreliosis in Denmark 1985-1990. A prospective study of 187 patients with Borrelia burgdorferi specific intrathecal antibody production. Brain. 1992;115(Pt 2):399-423. http://dx.doi.org/10.1093/brain/115.2.399 PMID:1606475

9. Hansen K. Lyme neuroborreliosis: improvements of the laboratory diagnosis and a survey of epidemiological and clinical features in Denmark 1985-1990. Acta Neurol Scand Suppl. 1994;151:1-44. PMID:8165888

10. Wilske B, Fingerle V, Schulte-Spechtel U. Microbiological and serological diagnosis of Lyme borreliosis. FEMS Immunol Med Microbiol. 2007;49(1):13-21. http://dx.doi.org/10.1111/j.1574695X.2006.00139.x PMID:17266710

11. Dessau RB, Bangsborg JM, Ejlertsen T, Skarphedinsson S, Schønheyder HC. Utilization of serology for the diagnosis of suspected Lyme borreliosis in Denmark: survey of patients seen in general practice. BMC Infect Dis. 2010;10(1):317. http:// dx.doi.org/10.1186/1471-2334-10-317 PMID:21040576

12. Voldstedlund M, Haarh M, Mølbak K, the MiBa Board of Representatives C; MiBa Board of Representatives. The Danish Microbiology Database (MiBa) 2010 to 2013. Euro Surveill. 2014;19(1):20667. http://dx.doi.org/10.2807/1560-7917. ES2014.19.1.20667 PMID:24434175

13. Voldstedlund M, Haahr M, Emborg HD, Bang H, Krause T. Real-time surveillance of laboratory confirmed influenza based on the Danish microbiology database (MiBa). Stud Health Technol Inform. 2013;192:978. 10.3233/978-1-61499-289-9-978 PMID:23920752

14. R: A language and environment for statistical computing [computer program]. Vienna, Austria: R Foundation for Statistical Computing; 2013.

15. Henningsson AJ, Christiansson M, Tjernberg I, Löfgren S, Matussek A. Laboratory diagnosis of Lyme neuroborreliosis: a comparison of three CSF anti-Borrelia antibody assays. Eur J Clin Microbiol Infect Dis. 2014;33(5):797-803. http://dx.doi. org/10.1007/s10096-013-2014-6 PMID:24263552

16. Berglund J, Eitrem R, Ornstein K, Lindberg A, Ringér A, Elmrud $\mathrm{H}$, et al. An epidemiologic study of Lyme disease in southern Sweden. N Engl J Med. 1995;333(20):1319-27. http://dx.doi. org/10.1056/NEJM199511163332004 PMID:7566023

17. Huppertz HI, Böhme M, Standaert SM, Karch H, Plotkin SA. Incidence of Lyme borreliosis in the Würzburg region of Germany. Eur J Clin Microbiol Infect Dis. 1999;18(10):697-703. http://dx.doi.org/10.1007/s100960050381 PMID:10584895 
18. Stanek G, Strle F. Lyme disease: European perspective. Infect Dis Clin North Am. 2008;22(2):327-39, vii. http://dx.doi. org/10.1016/j.idc.2008.01.001 PMID:18452805

19. Suppli $\mathrm{CH}$, Valenthiner-Branth $\mathrm{P}$, Hoffmann S. Purulent meningitis 2013. EPI-NYT 2014;(41).

20. Mulder S, van Vliet AJ, Bron WA, Gassner F, Takken W. High risk of tick bites in Dutch gardens. Vector Borne Zoonotic Dis. 2013;13(12):865-71. http://dx.doi.org/10.1089/vbz.2012.1194 PMID:24107214

21. Carlsson SA, Granlund H, Nyman D, Wahlberg P; Sten-

Anders Carlsson, Hans Granlund. IgG seroprevalence of

Lyme borreliosis in the population of the Aland Islands in Finland. Scand J Infect Dis. 1998;30(5):501-3. http://dx.doi. org/10.1080/00365549850161520 PMID:10066053 